\title{
Percepción de los Estudiantes de Anatomía Humana Frente a un Método de Enseñanza y Aprendizaje Basado en la Construcción de un Modelo de Pelvis
}

\author{
Perception of Human Anatomy Students Facing a Learning and \\ Teaching Methodology Based on the Construction of a Pelvis Model
}

\author{
Samuel Núñez-Cook ${ }^{1}$; Paola Gajardo²; Pablo A. Lizana ${ }^{1}$; Gustavo Vega-Fernandez ${ }^{1}$; \\ Alonso Hormazabal-Peralta ${ }^{1}$ \& Octavio Binvignat $^{1}$
}

NÜÑEZ-COOK, S.; GAJARDO, P.; LIZANA, P.A. VEGA-FERNANDEZ, G.; HORMAZABAL-PERALTA, A. \& BINVIGNAT, O. Percepción de los estudiantes de anatomía humana frente a un método de enseñanza y aprendizaje basado en la construcción de un modelo de pelvis. Int. J. Morphol., 36(1):221-225, 2018.

RESUMEN: Este estudio describe la percepción de estudiantes de anatomía humana frente a la actividad de construcción de modelos tridimensionales (3-D) a escala de pelvis. Pocos estudios evalúan la percepción de los estudiantes frente a una actividad de enseñanza y aprendizaje con modelos 3-D. La actividad se aplicó a un curso de anatomía humana de la carrera de obstetricia y puericultura a los cuales se les entregó modelos de estructuras óseas de la zona pélvica a escala real para que incorporaran las estructuras anatómicas (ligamentos, músculos, vascularización e inervación), finalizando la actividad se les entregó un cuestionario de percepción. 60 estudiantes realizaron la actividad de construcción de modelo anatómico 3-D y además completaron el cuestionario. Se reportaron niveles mayores al $93 \%$ de estudiantes que sintieron motivación, creen que fue un facilitador del aprendizaje y que la actividad fue bien planificada. Entre los aspectos negativos se registraron: el tiempo de trabajo y los costos involucrados. Dada la positiva percepción de los estudiantes frente a las actividades de construcción de modelos anatómicos, se considera que es una metodología adecuada dentro de los procesos de enseñanza y aprendizaje de la anatomía humana.

PALABRAS CLAVE: Educación médica; Anatomía humana; Modelo 3-d, Enseñanza y aprendizaje; Obstetricia.

\section{INTRODUCCIÓN}

La anatomía humana es la ciencia más antigua que posee la historia de la educación médica formal y a la vez ha mantenido su importancia pese a las modificaciones que ha sufrido en base a convenciones de nomenclatura, terminología e incluso comprensión de esta misma (Babinski et al., 2003; Hadie et al., 2014). Del mismo modo la enseñanza de esta ciencia también se ha visto modificada, reflejándose en situaciones como las prácticas con cadáveres, pues aunque profesionales y estudiantes las consideran importantes para el logro del aprendizaje, actualmente existe una escasez de cadáveres para la enseñanza mediante disección tradicional (Babinski et al.; Collipal \& Silva, 2011; Hadie et al.). Cabe destacar que el contexto tampoco es favorable, debido a que existe una disminución notoria en el contenido y número de horas para la enseñanza que se imparten en los cursos de anatomía basados en reformas de los planes de estudios médicos (Inzunza et al. 2003; Hadie et al.) y específicamente en Chile existe un aumento en el número de estudiantes por clase, lo cual genera una relación profesor / estudiante bastante preocupante (Lizana et al., 2015). Por lo tanto se han implementado nuevos métodos de enseñanza, como lo es el uso de software de simulación, módulos de autoaprendizaje, imágenes anatómicas computacionales, videos, entre otros métodos de enseñanza. El problema de estos métodos es que no logran abordar una concepción tridimensional (3-D) de la estructura anatómica adecuadamente (Collipal \& Silva), sin embargo una posible solución para el aprendizaje de esto es el uso de modelos anatómicos 3-D (Collipal \& Silva; Lizana et al.; Contreiras, 2013). Los modelos son 'reemplazantes' de los sistemas reales en estudio que son complejos para ser comprendidos en su totalidad, siendo, por lo tanto, representaciones que conservan las características fundamentales, actuando así como 'catalizadores' de la comprensión del mundo real (Lizana et al.). Las ventajas 
del uso de modelos 3-D en la enseñanza podrían incrementar cuando la actividad implica el uso de habilidades espaciales y creativas, como es el caso de la construcción de un modelo anatómico en tamaño real por parte de los estudiantes, pues el aumento en la carga cognitiva, la integración de un proceso asociado a conocimiento anatómico y la manipulación tangible pueden ser factores críticos para el éxito con el aprendizaje 3-D (Lizana et al.). Si se compara con otras metodologías los modelos físicos 3-D parecen ser una herramienta práctica para elevar el nivel de conocimientos de anatomía debido a sus ventajas para enseñar los arreglos espaciales de los órganos anatómicos y además su fácil accesibilidad dado a su bajo costo (Yammine \& Violato, 2016). Casos como la implementación de este tipo de actividad para la enseñanza del aparato locomotor (Lizana et al.) o en neuroanatomía (Estevez et al., 2010) han demostrado un rendimiento significativamente mejor en los estudiantes. Este tipo de actividad puede ser fundamental para las nuevas metodologías en la enseñanza de la anatomía humana en la educación médica y por lo tanto se deberían realizar más estudios al respecto. Las profesiones médicas por lo general tienen un área de especialización, la cual necesita profundizar ciertos conocimientos por sobre otros. En el caso de la obstetricia, las regiones y estructuras asociadas a la pelvis son uno de los conocimientosanatómicos esenciales según la International Confederation of Midwifery (2013), por esto, su enseñanza debe buscar las posibilidades del mejor aprendizaje y un factor importante para un buen aprendizaje es la percepción del estudiante frente a la actividad, de hecho, es el punto de inicio de toda gestión de calidad en procesos educativos (RemuzgoArtezano, 2008). Tomando en cuenta que la actividad de construcción de un modelo anatómico tamaño real de pelvis puede ser una alternativa para lograr un buen aprendizaje en obstetricia, nuestro objetivo de estudio es evaluar la percepción de los estudiantes de la carrera de obstetricia y puericultura frente al trabajo de construcción de modelo de pelvis a partir de réplicas óseas a escala.

\section{MATERIAL Y MÉTODO}

Participantes: El estudio se realizó en el curso de anatomía aplicada correspondiente a la carrera de obstetricia y puericultura durante el segundo semestre de 2016 (agosto a diciembre) en la Universidad Autónoma de Chile, sede Talca. El curso se basó en anatomía específica de obstetricia (dedicada a pelvis), el cual tenía una cantidad total de 92 estudiantes divididos en grupos de laboratorios de máximo 30. Criterio para la inclusión al estudio fue haber asistido a todas las sesiones de la actividad y haberla desarrollado, mientras que la etapa de encuesta fue anónima.
Actividad de construcción del modelo: Los estudiantes recibieron muestras de yeso dental de piezas óseas humana femeninas de la región pélvica obtenidas a partir de moldes de caucho silicona realizados en base del protocolo utilizado por Lizana et al., con huesos reales, esto es: coxal derecho, coxal izquierdo, sacro y coxis.

A partir de las réplicas los estudiantes debieron construir el modelo anatómico de pelvis femenina. Primero identificaron y describieron las estructuras basándose en la terminología anatómica internacional, alcanzada esta meta se articulan los diferentes huesos para conformar una pelvis ósea. Se procede al estudio de ejes, planos y divisiones del conjunto (pelvimetría externa e interna).

Posteriormente, realizan los procedimientos para construir los ligamentos de las articulaciones de la pelvis, respetando sus características principales. Se continúa con el montaje y construcción de los músculos, observando orígenes e inserciones de los elementos musculares y sus acciones. Se prosigue con la preparación de la vascularización. Por último, se construyen los nervios y su distribución.

Para la construcción personalizada de cada pelvis, los estudiantes también utilizan una serie de recursos de apoyo como: piezas cadavéricas disecadas, modelos anatómicos, simuladores, imágenes en 3-D, orientaciones y sugerencias de los tutores, monitores y profesores, incluyendo una síntesis escrita de la pelvis femenina. Evaluaciones sumativas se van realizando durante todos los periodos (datos no reportados en este trabajo).

Cuestionario: Luego de desarrollada la actividad de construcción de modelo anatómico 3-D por parte de los estudiantes, se administró una encuesta de percepción que incluyó diferentes preguntas (abiertas y cerradas). La encuesta fue completamente anónima y se refirió a la motivación frente a la actividad, dificultad, importancia para el aprendizaje, aspectos negativos y positivos de ella, sugerencias, y características principales de la actividad, todo en base a lo que percibió el estudiante.

Análisis estadístico: Las respuestas de los estudiantes de obstetricia y puericultura que contestaron a las preguntas fueron reportadas como frecuencia (n) y porcentaje (\%), para esto se utilizó el programa STATA 12.0 para Windows.

\section{RESULTADOS}

Se respondieron de forma completa 60 cuestionarios $\mathrm{y}$ todos los estudiantes terminaron el trabajo con el modelo 


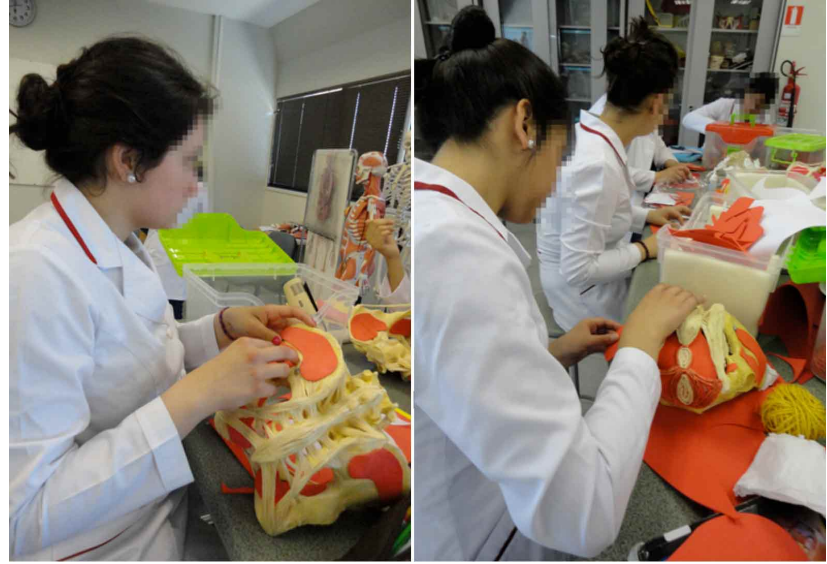

Fig. 1. Estudiantes de obstetricia de la asignatura de anatomía humana durante el proceso de construcción del modelo de pelvis.

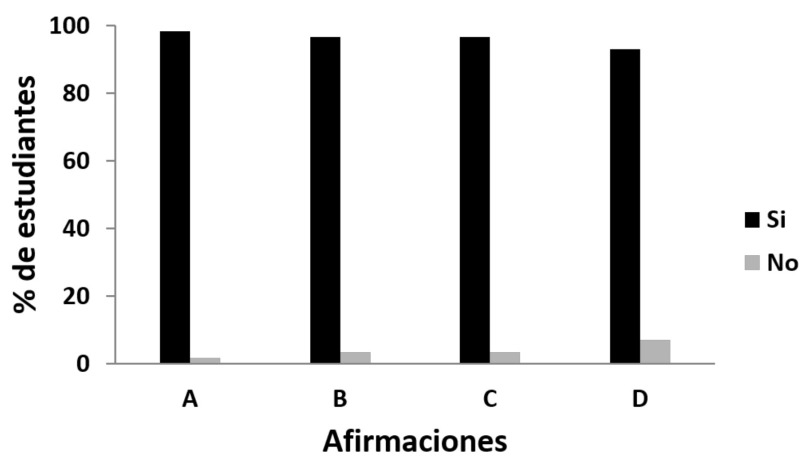

Fig. 2. Afirmaciones de los estudiantes frente al trabajo con modelos de pelvis $(n=60)$. A: si sintió motivación con la actividad. B: si considera la actividad como un facilitador de su aprendizaje. C: si considera que la actividad fue bien planificada. D: si va a conservar el modelo construido.

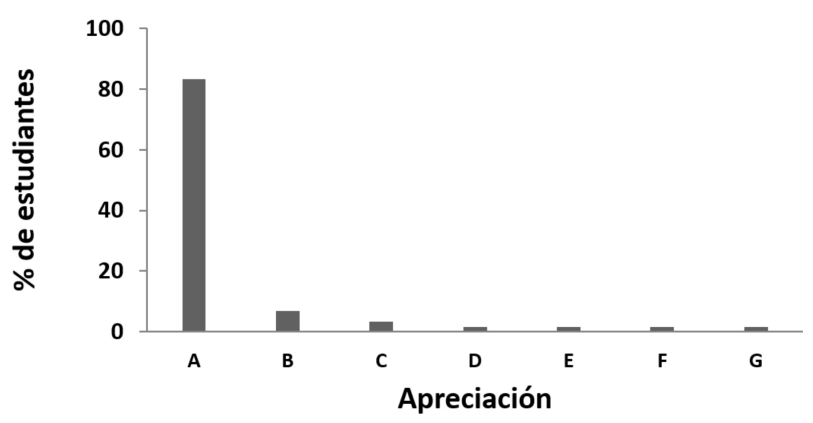

Fig. 3. Apreciación de la actividad con modelos anatómicos de pelvis por parte de los estudiantes $(n=60)$. A: Combinación de ser muy interesante, muy aplicable, muy útil y muy organizado. B: Solo muy útil. C: Combinación de ser escasamente interesante, aplicable, útil y organizado. D: Combinación de ser interesante, útil, muy aplicable y organizado. E: Solo muy interesante y muy útil. F: Combinación de ser interesante, muy útil, aplicable, escasamente organizado. G: Solo muy interesante. de pelvis (Fig.1). Del cuestionario, una alta mayoría de la muestra sintió motivación con la actividad, además creen que resultó ser un facilitador para su aprendizaje, y les parece bien planificada, todos estos resultados sobre el $93 \%$ (Fig. 2). Además, la mayoría de los estudiantes apreció que los tiempos asignados para actividades de laboratorios, interrogación y auto-aprendizaje de esta metodología fueron adecuados $(93,33 \%, 100 \%, 93,33 \%$ respectivamente). En relación al nivel de dificultad con un 93,1 \% los estudiantes consideran que el nivel de dificultad de la actividad es medio. En lo que respecta a la apreciación de la actividad el $83,15 \%$ de los estudiantes clasificó la actividad en conjunto como: muy interesante, muy aplicable, muy útil, y bien organizada (Fig. 3).

Ante la pregunta si aprendieron después de la actividad, el 42,37 \% dijo que aprendió lo esperado, el 57,63\% sostuvo que incluso más y ninguno de los estudiantes indicó que aprendió menos de lo esperado. Al referirse sobre los aspectos de la actividad que gustaron o no a los estudiantes, el 53,33\% de los ellos indicó que les gustó la metodología y su didáctica, siguiendo la aplicabilidad, el resto de los aspectos como la convivencia y el aprendizaje que entrega fueron mencionados por sumas menores al $10 \%$ de los estudiantes, niveles bajo el $2 \%$ indicó que no le gustó ninguno (Fig. 4). Entre los aspectos que no gustaron, el mayor porcentaje se encuentra en los gastos, siguiéndole el tiempo entregado, añadiendo que un 11,67 \% mencionó ambos, también se nombró el no uso de estructuras reales, interrogaciones y contenidos utilizados e incluso la ponderación de la actividad, pero todas ellas con niveles inferiores al $10 \%$ (Fig. 5). Cuando se les consultó por propuestas de mejoras, más de la mitad sostuvo que no necesitaba mejoras la actividad, mientras que las propuestas mencionadas mantienen niveles parecidos de porcentaje (alrededor del $10 \%$ ), siendo éstas: tiempo, el incorporar musculatura en la evaluación, y agregar otras estructuras como la región perineal.

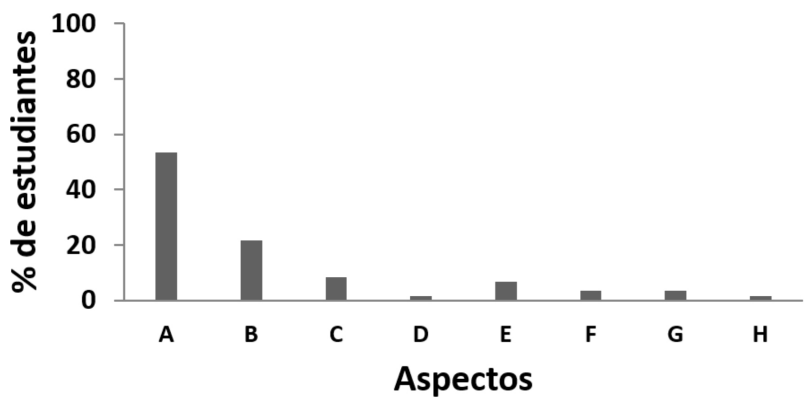

Fig. 4. Aspectos positivos de la actividad considerados por los estudiantes (n=60). A: Metodología y didáctica. B: Aplicabilidad. C: Aprendizaje. D: Convivencia. E: Metodología y convivencia. F: Metodología y aprendizaje. G: Aprendizaje y convivencia. H: Ningún aspecto. 


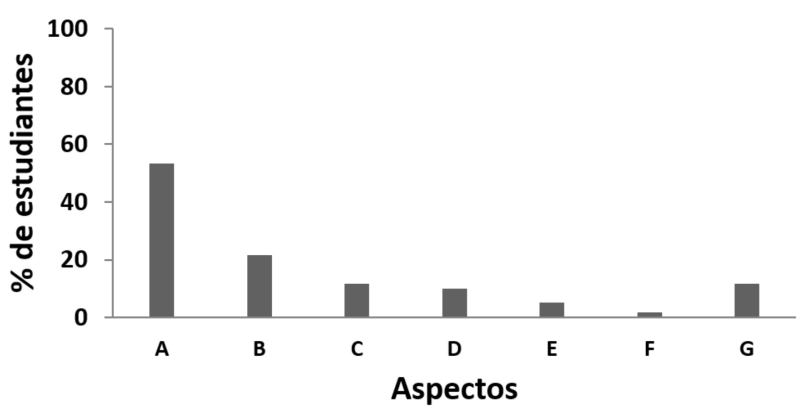

Fig. 5. Aspectos negativos de la actividad por parte de los estudiantes frente al trabajo con modelos anatómicos de pelvis $(\mathrm{n}=60)$. A: Gastos. B: Tiempo. C: Tiempo y gastos. D: Contenido e interrogación. E: Sin estructuras reales. F: Ponderación. G: Ningún aspecto.

\section{DISCUSIÓN}

En este estudio se evaluó la percepción de estudiantes de obstetricia y puericultura frente a una actividad diseñada para enseñar anatomía humana de la región pélvica utilizando un modelo óseo en 3-D en escala real para la construcción y ubicación de las estructuras anatómicas. El principal hallazgo del estudio es que los estudiantes de obstetricia tienen una buena percepción de la actividad, resultados que concuerdan con estudios similares con estudiantes de pregrado, como por ejemplo en laboratorios de cursos de neurociencia de estudiantes de medicina donde se indica una clara preferencia por este tipo de actividad (Estevez et al.), también en profesores de biología en formación donde indican que este tipo de intervenciones promueven su motivación por el curso de anatomía y por su aprendizaje (Lizana et al.). Además, en esta investigación los estudiantes de obstetricia describen que la actividad facilitó su aprendizaje, esto puede deberse al desarrollo de habilidades espaciales (Huk et al., 2006) señalando que las actividades basadas en construcción de modelos anatómicos 3-D promueven el aprendizaje, elemento que se podría relacionar si consideramos que los modelos 3-D favorecen el aprendizaje anatómico generando un puente eficaz entre las estructuras 2-D y 3-D (Lizana et al.). Estudios similares utilizando arcilla para la construcción de modelos anatómicos muestran un aumento significativo en el aprendizaje de los estudiantes de medicina, resultando ser una actividad eficaz para la enseñanza y aprendizaje de estructuras anatómicas en cortes transversales (Oh et al., 2009), también la comprensión de estructuras perivetriculares del cerebro ha sido significativamente mejorada utilizando la construcción de modelos de arcilla con estudiantes de medicina (Akle et al., 2017). Otros estudios realizados con estudiantes de obstetricia demuestran que una intervención de modelamientos anatómicos de arcilla de la zona pélvica durante el programa de clases presentan una eficacia significativa para el aprendizaje de los estudiantes (Myers et al., 2001; Schmidt et al., 2017). Nuestros resultados pueden estar relacionados con los de los estudios mencionados anteriormente, dado que la percepción de los estudiantes frente a una actividad es un factor importante en el aprendizaje obtenido (Remuzgo-Artezano) y como la percepción de los estudiantes frente a la actividad de construcción de modelos anatómicos 3-D es descrita como positiva y buena, es lógico inferir que la actividad mejore el aprendizaje de los alumnos como se ha observado en diversos estudios.

Los estudiantes caracterizan también la construcción de modelos anatómicos como una actividad muy interesante, útil para el desempeño profesional y aplicable, propiedades que en otros estudios, como Estevez et al. y Lizana et al., se aprecian. Estudiantes de kinesiología y fonoaudiología también valoran positivamente este tipo de actividad en López et al. (2011) donde también se menciona que una característica que se puede observar en este tipo de actividades es la disminución del estrés y ansiedad por parte de los estudiantes, esto podría estar relacionado con el efecto en la percepción positiva que se tiene frente a la actividad. La mayoría de los estudiantes indicaron que el nivel de dificultad que aprecian de esta metodología fue media, siendo teóricamente lo recomendable, pues una complejidad alta (que conlleva una sobrecarga cognitiva), como también una muy simple, puede afectar el buen desarrollo del objetivo y aprendizaje de una actividad, ya que es un factor importante en la perspectiva del estudiante y por ende, en su motivación (Mitchell \& Carbone, 2011).

Se pudieron rescatar algunas críticas constructivas, una de ellas es la inclusión de estructuras musculares en la evaluación, que se infiere como una necesidad de aprendizaje por parte de los estudiantes de obstetricia, mayores estudios se necesitan en los procesos de evaluación.

Los aspectos de la actividad que tuvieron rechazo por parte de los estudiantes fueron de carácter externa, ya que señalan un descontento con los gastos utilizados en ella (compra y traslado) y no de la metodología propiamente tal, lo que sugiere la búsqueda de soluciones frente a este caso, tales como la implementación de materiales de la organización educativa u otros. Un aspecto que muestra incongruencia de los estudiados es la perspectiva que tienen frente al tiempo utilizado durante el proceso, en primera instancia la mayoría declara pensar que el tiempo utilizado es el adecuado, mientras que en una segunda oportunidad indican que es una de las cosas que menos gustaron, por lo tanto es necesario profundizar en estudios con respecto a este tema. Sin embargo, como se ha señalado anteriormente la vista general frente a la actividad es muy positiva. En este sentido, la visión general de los estudiantes es que la actividad fue bien organizada y planificada 
en su desarrollo, otro aspecto que teóricamente es ampliamente recomendado para actividades de procesos de construcción de modelos científicos para la enseñanza (Justi, 2006).

Este es un estudio transversal, es decir, que permite levantar datos de un solo momento, es por esto, que se sugiere realizar un seguimiento para esta propuesta metodológica que permita obtener información complementaria a través del tiempo, Además, el estudio se realizó en estudiantes de una sola universidad, debido a las posibilidades de implementación del estudio para los investigadores, por eso incentivamos su aplicación a una escala mayor o en otras universidades para enriquecer los conocimiento respecto a este tema.

En resumen, la percepción de los estudiantes de obstetricia frente a la actividad de construcción de un modelo anatómico de la región pélvica es bastante positiva, caracterizada como muy útil, interesante y favorecedor del aprendizaje. Los aspectos a mejorar son de carácter externo como el origen de los materiales a utilizar o las dificultades del transporte. Dada la positiva percepción del estudiante frente a las actividades de construcción de modelos anatómicos, consideramos que es una metodología adecuada dentro de los procesos de enseñanza y aprendizaje de la anatomía. Por otro lado, se estima que se debe seguir investigando en la evaluación de los aprendizajes de los estudiantes en comparación con otras metodologías.

NUÑEZ-COOK, S.; GAJARDO, P.; LIZANA, P. A.; VEGAFERNANDEZ，G.; HORMAZABAL-PERALTA，A. \& BINVIGNAT, O. Perception of human anatomy students facing a learning and teaching methodology based on the construction of a pelvis model. Int. J. Morphol., 36(1):221-225, 2018.

SUMMARY: In this study, the perception of human anatomy students faced with constructing a three-dimensional (3-D) pelvis model (to scale) was studied. Few studies evaluate the perception of students facing learning and teaching methodologies with 3-D models. The activity was performed with students from a human anatomy course as part of the obstetrics degree, in which models of pelvic zone bone structures (to scale) were supplied in order to incorporate the anatomic structures (ligaments, muscles, vascularization and innervation). Once the activity was finished, a perception questionnaire was taken. 60 students participated in the activity of constructing the 3-D anatomical model and also completed the questionnaire. It was found that over $93 \%$ of the students felt motivated, believed the activity facilitated learning and also that the activity was well planned. Some of the negative factors that were reported included time taken and the associated costs. Due to the positive perception of the students involved in the activity of constructing anatomical models, this is considered to be a suitable methodology for use during the process of teaching and learning about human anatomy.

KEY WORDS: Medical education; Human anatomy; 3D models; Learning and teaching; Obstetrics.

\section{REFERENCIAS BIBLIOGRÁFICAS}

Akle, V.; Peña-Silva, R.; Valencia, D. M. \& Rincón-Perez, C. W: Validation of clay modeling as a learning tool for the periventricular structures of the human brain. Anat. Sci. Educ., 2017. doi: 10.1002/ase.1719. [Epub ahead of print].

Babinski, M. A. Sgrott, E. A.; Luz, H. P.; Brasil, F. B.; Chagas, M. A. \& AbiduFigueiredo, M. The relationship of the students with corpse in the practical study of anatomy: the reaction and influence in the learning. Int. J. Morphol., 21(2):137-42, 2003.

Collipal, L. E. \& Silva, M. H. Study of anatomy in cadavers and anatomical models. Impression of students. Int. J. Morphol., 29(4):1181-5, 2011.

Contreiras, N. O Ensino e o Aprendizado Práticos da Anatomia Humana: Uma Revisão de Literatura. Monografía. Salvador de Bahia, Universidade Federal da Bahia, Faculdade de Medicina da Bahia, 2013.

Estevez, M. E.; Lindgren, K. A. \& Bergethon, P. R. A novel three-dimensional tool for teaching human neuroanatomy. Anat. Sci. Educ., 3(6):309-17, 2010.

Hadie, S. N. H.; Hassan, A., Ismail, Z. I. M.; Asari, M. A.; Khan, A. A.; Kasim, F.; Yusof, N. A. M.; Sulong, H. A. M.; Muda, T. F. M. T.; Arifin, W. N. \& Yusoff, M. S. B. Developing constructs of anatomy education environment measurement: A delphi study. Procedia Soc. Behav. Sci., 116:4219-23, 2014.

Huk, T. Who benefits from learning with 3D models? The case of spatial ability. J. Comput. Assist. Learn., 22(6):392-404, 2006.

International Confederation of Midwifery. Global standards of Midwifery Education 2010, Amended 2013. Amsterdam, ICM, 2013.

Inzunza, O.; D'Acuña, E. \& Bravo, H. Practical evaluation in anatomy. Performance of first year medical students in relation to different ways of asking questions. Int. J. Morphol., 21(2):131-6, 2003.

Justi, R. La enseñanza de ciencias basada en la elaboración de modelos. Enseñ. Cienc., 24(2):173-84, 2006.

Lizana, P. A.; Merino, C.; Bassaber, A.; Henríquez, R.; Vega-Fernández, G. \& Binvignat, O. Learning human anatomy using three-dimensional models made from real-scale bone pieces: Experience with the knee joint among pre-service biology teachers. Int. J. Morphol., 33(4):1299-306, 2015.

López, F. B.; Sandoval, M. C.; Giménez, M. A. M. \& Rosales, V. P. Assessment of anatomical models activity in the competence development in undergraduate students and their relationship to learning style, career and sex. Int. J. Morphol., 29(2):568-74, 2011.

Mitchell, I. \& Carbone, A. A typology of task characteristics and their effects on student engagement. Int. J. Educ. Res., 50(5-6):257-70, 2011.

Myers, D. L.; Arya, L. A.; Verma, A.; Polseno, D. L. \& Buchanan, E. M. Pelvic anatomy for obstetrics and gynecology residents: an experimental study using clay models. Obstet. Gynecol., 97(2):321-4, 2001.

Oh, C. S.; Kim, J. Y. \& Choe, Y. H. Learning of cross-sectional anatomy using clay models. Anat. Sci. Educ., 2(4):156-9, 2009.

Remuzgo-Artezano, A. Percepción de los alumnos respecto al proceso enseñanza-aprendizaje en el programa de segunda especialización de enfermería en la UNMSM - 2008. Tesis para optar el grado académico de Magister en Docencia e Investigación en Salud. Lima, Universidad Nacional Mayor de San Marcos, 2010.

Schmidt, B.; Thompson, B. J. \& Chang, A. The use of clay models to teach pelvic anatomy to first and second year medical students at OUWB School of Medicine. FASEB J., 31(1 Suppl.):582.1, 2017

Yammine, K \& Violato, C. The effectiveness of physical models in teaching anatomy: a meta-analysis of comparative studies. Adv. Health Sci. Educ. Theory Pract., 21(4):883-95, 2016.

\section{Dirección para correspondencia:}

Dr. Octavio Binvignat Gutiérrez

Laboratorio de Ciencias Morfológicas

Instituto de Biología

Pontificia Universidad Católica de Valparaíso

Avda. Universidad 330

Valparaíso - CHILE

E-mail. binvigna@terra.com.br

Recibido: 27-08-2017

Aceptado: 23-12-2017 